\title{
Evidence of Lensing of the Cosmic Microwave Background by Dark Matter Halos
}

Mathew Madhavacheril, ${ }^{1}$ Neelima Sehgal, ${ }^{1}$ Rupert Allison, ${ }^{2}$ Nick Battaglia, ${ }^{3}$ J. Richard Bond, ${ }^{4}$ Erminia Calabrese, ${ }^{2}$ Jerod Caligiuri, ${ }^{5}$ Kevin Coughlin, ${ }^{6}$ Devin Crichton, ${ }^{7}$ Rahul Datta, ${ }^{6}$ Mark J. Devlin, ${ }^{8}$ Joanna Dunkley, ${ }^{2}$ Rolando Dünner, ${ }^{9}$ Kevin Fogarty, ${ }^{7}$ Emily Grace, ${ }^{10}$ Amir Hajian, ${ }^{4}$ Matthew Hasselfield, ${ }^{11}$ J. Colin Hill, ${ }^{12}$ Matt Hilton, ${ }^{13}$ Adam D. Hincks, ${ }^{14}$ Renée Hlozek, ${ }^{11}$ John P. Hughes, ${ }^{15}$ Arthur Kosowsky, ${ }^{5}$ Thibaut Louis, ${ }^{2}$ Marius Lungu, ${ }^{8}$ Jeff McMahon, ${ }^{6}$

Kavilan Moodley, ${ }^{13}$ Charles Munson, ${ }^{6}$ Sigurd Naess, ${ }^{2}$ Federico Nati, ${ }^{16}$ Laura Newburgh, ${ }^{17}$ Michael D. Niemack, ${ }^{18}$ Lyman A. Page, ${ }^{10}$ Bruce Partridge, ${ }^{19}$ Benjamin Schmitt, ${ }^{8}$ Blake D. Sherwin, ${ }^{20}$ Jon Sievers, ${ }^{21,22}$ David N. Spergel, ${ }^{11}$ Suzanne T. Staggs, ${ }^{10}$ Robert Thornton, ${ }^{23,8}$ Alexander Van Engelen, ${ }^{4}$ Jonathan T. Ward, ${ }^{8}$ and Edward J. Wollack ${ }^{24}$

(Atacama Cosmology Telescope Collaboration)

\author{
${ }^{1}$ Physics and Astronomy Department, Stony Brook University, Stony Brook, New York 11794, USA \\ ${ }^{2}$ Sub-Department of Astrophysics, University of Oxford, Keble Road, Oxford OXI 3RH, United Kingdom \\ ${ }^{3}$ McWilliams Center for Cosmology, Carnegie Mellon University, Department of Physics, 5000 Forbes Ave., Pittsburgh, \\ Pennsylvania 15213, USA \\ ${ }^{4}$ Canadian Institute for Theoretical Astrophysics, University of Toronto, Toronto, Ontario, Canada M5S $3 H 8$ \\ ${ }^{5}$ Department of Physics and Astronomy, University of Pittsburgh, Pittsburgh, Pennsylvania 15260, USA \\ ${ }^{6}$ Department of Physics, University of Michigan, Ann Arbor, Michigan 48103, USA \\ ${ }^{7}$ Department of Physics and Astronomy, The Johns Hopkins University, 3400 N. Charles St., Baltimore, Maryland 21218-2686, USA \\ ${ }^{8}$ Department of Physics and Astronomy, University of Pennsylvania, 209 South 33rd Street, Philadelphia, Pennsylvania 19104, USA \\ ${ }^{9}$ Departamento de Astronomía y Astrofísica, Pontificía Universidad Católica, Casilla 306, Santiago 22, Chile \\ ${ }^{10}$ Joseph Henry Laboratories of Physics, Jadwin Hall, Princeton University, Princeton, New Jersey 08544, USA \\ ${ }^{11}$ Department of Astrophysical Sciences, Peyton Hall, Princeton University, Princeton, New Jersey 08544, USA \\ ${ }^{12}$ Department of Astronomy, Pupin Hall, Columbia University, New York, New York 10027, USA \\ ${ }^{13}$ Astrophysics and Cosmology Research Unit, School of Mathematics, Statistics and Computer Science, \\ University of KwaZulu-Natal, Durban 4041, South Africa \\ ${ }^{14}$ Department of Physics and Astronomy, University of British Columbia, Vancouver, British Columbia, Canada V6T 1Z4 \\ ${ }^{15}$ Department of Physics and Astronomy, Rutgers, The State University of New Jersey, Piscataway, New Jersey 08854-8019, USA \\ ${ }^{16}$ Dipartimento di Fisica, Università La Sapienza, P. le A. Moro 2, 00185 Roma, Italy \\ ${ }^{17}$ Dunlap Institute, University of Toronto, 50 St. George St., Toronto, Ontario, Canada M5S $3 H 4$ \\ ${ }^{18}$ Department of Physics, Cornell University, Ithaca, New York 14853, USA \\ ${ }^{19}$ Department of Physics and Astronomy, Haverford College, Haverford, Pennsylvania 19041, USA \\ ${ }^{20}$ Berkeley Center for Cosmological Physics, LBL and Department of Physics, University of California, Berkeley, \\ California 94720, USA \\ ${ }^{21}$ Astrophysics and Cosmology Research Unit, School of Chemistry and Physics, University of KwaZulu-Natal, \\ Durban 4041, South Africa \\ ${ }^{22}$ National Institute for Theoretical Physics (NITheP), University of KwaZulu-Natal, Private Bag X54001, Durban 4000, South Africa \\ ${ }^{23}$ Department of Physics, West Chester University of Pennsylvania, West Chester, Pennsylvania 19383, USA \\ ${ }^{24}$ NASA/Goddard Space Flight Center, Greenbelt, Maryland 20771, USA
}

(Received 30 November 2014; revised manuscript received 13 February 2015; published 13 April 2015; publisher error corrected 17 April 2015)

We present evidence of the gravitational lensing of the cosmic microwave background by $10^{13}$ solar mass dark matter halos. Lensing convergence maps from the Atacama Cosmology Telescope Polarimeter (ACTPol) are stacked at the positions of around 12000 optically selected CMASS galaxies from the SDSS-III/BOSS survey. The mean lensing signal is consistent with simulated dark matter halo profiles and is favored over a null signal at $3.2 \sigma$ significance. This result demonstrates the potential of microwave background lensing to probe the dark matter distribution in galaxy group and galaxy cluster halos.

DOI: 10.1103/PhysRevLett.114.151302

PACS numbers: 98.70.Vc, 95.35.+d, 98.62.Sb

Introduction.-Measuring the gravitational lensing of the cosmic microwave background (CMB) by intervening structure is a potentially powerful way to map out the mass distribution in the Universe. Advantages of CMB lensing over lensing measured at other wavelengths include that the CMB is a source that fills the whole sky, is at a known redshift, and has well understood statistical properties. To date, the lensing of the CMB caused by the large-scale 
projected dark matter distribution has been observed by a number of $\mathrm{CMB}$ experiments with ever increasing statistical significance [1-5]. This lensing signal has been detected in both CMB temperature and polarization maps and in cross-correlation with other tracers of large-scale structure [1,2,5-18]. These CMB lensing measurements have become precise enough that they now provide interesting constraints on a number of cosmological parameters such as curvature and the amplitude of matter fluctuations [19]. These constraints can be expected to significantly improve with the advent of near-term and next-generation CMB data sets [20-22].

Previous studies have focused on the lensing of the CMB by large-scale structure corresponding to scales between tens and several hundred comoving Mpc. As the data improve, it is possible to shift focus to smaller scales, particularly those which have undergone appreciable nonlinear growth. On small enough scales, the CMB is lensed by individual dark matter halos. We refer to this small-scale signal as "CMB halo lensing," and note that this lensing can be due to individual galaxy clusters, galaxy groups, and massive galaxies. Before now, CMB experiments did not have the sensitivity or resolution to detect this signal which was hypothesized to exist over a decade ago [23-35].

In this Letter, we present evidence of the $\mathrm{CMB}$ halo lensing signal using the first season of data from ACTPol. This detection is made by stacking ACTPol reconstructed convergence maps at the positions of CMASS galaxies that have been optically selected from the Sloan Digital Sky Survey-III Baryon Oscillation Spectroscopic Survey Tenth Data Release (SDSS-III/BOSS DR10) ([36-38]). This signal is detected at a significance of $3.2 \sigma$ when we combine the nighttime data from three ACTPol first-season survey regions. We see an excess of $1.3 \sigma$ or greater in each individual survey region, although all fields are needed to give a statistical detection.

CMB Data.-ACT is located in Parque Astronómico Atacama in northern Chile at an altitude of $5190 \mathrm{~m}$. The 6-meter primary mirror has a resolution of 1.4 arcminutes at a wavelength of 2 millimeters. Its first polarization-sensitive camera, ACTPol, is described in detail in [39] and [40]. ACTPol observed from Sept. 11 to Dec. 14, 2013 at $146 \mathrm{GHz}$. Four "deep field" patches were surveyed near the celestial equator at right ascensions of $150^{\circ}, 175^{\circ}, 355^{\circ}$, and $35^{\circ}$, which we call $D 1\left(73 \mathrm{deg}^{2}\right)$, D2 (70 deg 2$), D 5\left(70 \mathrm{deg}^{2}\right)$, and D6 (63 deg 2$)$. The scan strategy allows for each patch to be observed in a range of different parallactic angles while scanning horizontally, which aids in separating instrumental effects from celestial polarization. White noise map sensitivity levels for the patches are $16.2,17,13.2$, and $11.2 \mu \mathrm{K}$ arcmin, respectively, in temperature, with polarization noise levels higher by roughly $\sqrt{2}$. All patches were observed during nighttime hours for some fraction of the time. The nighttime data fraction is $50 \%, 25 \%, 76 \%$, and $94 \%$ for $D 1, D 2, D 5$, and
$D 6$, respectively. We use only nighttime data from $D 1, D 5$, and $D 6$ in this analysis. Further details about the observations and mapmaking can be found in [40].

We template-subtract point sources from these maps by filtering the $D 1, D 5$, and $D 6$ patches with a filter matched to the ACTPol beam profile. Point sources with a signal at least 5 times larger than the background uncertainty in the filtered maps are identified, and their fluxes are measured. A template of beam-convolved point sources is then constructed for each patch and subsequently subtracted from the corresponding patch. As a result, point sources with fluxes above $8 \mathrm{mJy}$ are removed from $D 1$, and sources with fluxes above $5 \mathrm{mJy}$ are removed from $D 5$ and $D 6$.

Overall, calibration of the ACTPol patches is achieved by comparing to the Planck $143 \mathrm{GHz}$ temperature map [41] and following the method described in [42]. The patches are then multiplied by a factor of 1.012 to correspond to the WMAP calibration as in [40].

Optical Data.-SDSS I and II obtained imaging data of $11000 \mathrm{deg}^{2}$ using the 2.5 -meter SDSS Telescope $[43,44]$. This survey has five photometric bands. SDSS-III BOSS extended this imaging survey by $3000 \mathrm{deg}^{2}$ [36]. Based on the resulting photometric catalog of galaxies, CMASS ("constant mass") galaxies were selected extending the luminous red galaxy (LRG) selection of [45] to bluer and fainter galaxies. These galaxies form a roughly volumelimited sample with $z>0.4$ and satisfy the criterion that their number density be high enough to probe large-scale structure at redshifts of about 0.5 [46]. The BOSS spectroscopic survey targeted these galaxies obtaining spectroscopic redshifts, and these galaxies have been used in a number of cosmological analyses [46,47].

Using the tenth SDSS public data release (DR10), we selected CMASS galaxies from the BOSS catalog [48]. This selection resulted in 6144, 5211, and 5420 CMASS galaxies that lie within $D 1, D 5$, and $D 6$, respectively. These galaxies span a redshift range of about $z=0.4$ to $z=0.7$, with a mean redshift of $z=0.54$. The galaxies were cross referenced with galaxies in the SDSS-III photometric catalog [49], using a shared galaxy identification number, to obtain more accurate celestial position information.

A subset of CMASS galaxies have optical weak-lensing mass estimates of their average halo masses using the publicly available CFHTLenS galaxy catalog $[50,51]$. This subset has an additional redshift cut of $z \in[0.47,0.59]$ and a stellar mass cut of $10^{11.1} h_{70}^{-2} M_{\odot}<M_{\star}<10^{12.0} h_{70}^{-2} M_{\odot}$ relative to the full CMASS sample. (The full CMASS sample has a stellar mass range of roughly $10^{10.6} h_{70}^{-2} M_{\odot}<$ $M_{\star}<10^{12.2} h_{70}^{-2} M_{\odot}$.) The average halo mass estimate for this CMASS galaxy subsample is $M_{200 \bar{\rho}_{0}}=(2.3 \pm 0.1) \times$ $10^{13} h^{-1} M_{\odot}$ [50], where $M_{200 \bar{\rho}_{0}}$ is defined as the mass within $R_{200}$, a radius within which the average density is 200 times the mean density of matter today. If we had adopted the additional redshift and stellar mass cuts of this subsample of CMASS galaxies, then the number of 
galaxies falling in the ACTPol patches would have been reduced by roughly a factor of 2; so we instead stack on the full CMASS galaxy sample within our survey regions for this Letter.

Since we cut out a $70^{\prime} \times 70^{\prime}$ "stamp" centered on each CMASS galaxy from the ACTPol temperature maps, we exclude all galaxies whose stamp does not fall entirely within the corresponding ACTPol patch. We find from simulations that this stamp size is roughly the minimum required to obtain unbiased lensing reconstructions using the pipeline described here. We also note that performing reconstructions on small stamps allows us to obtain the necessary precision for the mean field subtraction described in the next section. To avoid noisy parts of the ACTPol patches, we also remove galaxies for which the mean value of its corresponding inverse variance weight stamp is lower than $0.7,0.3$, and 0.3 times the mean of the weight map of the full patch for $D 1, D 5$, and $D 6$, respectively. These factors were chosen so that all of the stamps in our stacks had an average detector hit count above the same minimum value. These cuts leave 4400, 3665, and 4032 galaxies to stack on in $D 1, D 5$, and $D 6$, respectively.

Pipeline.-The analysis pipeline used in this Letter is as follows. We set the mean of each galaxy-centered $70^{\prime} \times 70^{\prime}$ stamp to zero to prevent leakage of power on scales larger than the stamp size due to windowing effects. Each stamp is then multiplied by an apodization window that consists of the corresponding inverse variance weight stamp that has been smoothed and tapered with a cosine window of width 14 arcminutes. Each of the stamps is then beam deconvolved and filtered with the quadratic filter given in [32].

The filter is constructed by noting that lensing of the $\mathrm{CMB}$ temperature field shifts the unlensed temperature field, $\tilde{T}(\hat{\mathbf{n}})$, to the lensed temperature field, $T(\hat{\mathbf{n}})$, so that

$$
T(\hat{\mathbf{n}})=\tilde{T}(\hat{\mathbf{n}}+\nabla \phi),
$$

where $\phi$ is the deflection potential and $\nabla \phi$ is the deflection angle. The lensing convergence, $\kappa$, is given by

$$
\nabla^{2} \phi=-2 \kappa .
$$

On the arcminute scales of individual dark matter halos, the unlensed $\mathrm{CMB}$ can be approximated as a gradient, and lensing induced by the halo alters the CMB field along this gradient direction. Thus, we search for this signal by looking for deflections correlated with the background CMB gradient. In order to do this, we reconstruct the lensing convergence field, $\kappa$, by constructing two filtered versions of the data: one that is filtered to isolate the background gradient and one that is filtered to isolate smallscale CMB fluctuations. Then, we take the divergence of the product of these two maps as described in [32] and summarized below.

The first filtered map is constructed by taking the weighted gradient of the lensed CMB map
$\mathbf{G}_{l}^{T T}=i l W_{l}^{T T} T_{l}$,

where the weight filter is

$$
W_{l}^{T T}=\tilde{C}_{l}^{T T}\left(C_{l}^{T T}+N_{l}^{T T}\right)^{-1}
$$

for $l \leq l_{\mathrm{G}}$, and $W_{l}^{T T}=0$ for $l>l_{\mathrm{G}}$. Note that $\tilde{C}_{l}$ and $C_{l}$ are the unlensed and lensed CMB power spectra, respectively, from a fiducial theoretical model based on Planck best-fit parameters, and $N_{l}$ is the noise power. Here, $l_{\mathrm{G}}$ is a cutoff scale and is set to $l_{\mathrm{G}}=2000$. We choose this cutoff since, as shown in [32], the unlensed CMB gradient does not have contributions above $l=2000$, and we want to remove smaller-scale fluctuations. This cutoff in the gradient filter is the main difference between the filter used in this Letter and the filter used for large-scale structure lensing [52]. When the convergence, $\kappa$, is large (of order 1 ), as it is for clusters, only the filter with the gradient cutoff returns an unbiased estimate of the convergence [32]. For smaller convergence values, as measured for galaxy groups in this work, both filters return similar results.

The second filtered map is an inverse-variance weighted map given by

$$
L_{l}^{T}=W_{l}^{T} T_{l},
$$

where

$$
W_{l}^{T}=\left(C_{l}^{T T}+N_{l}^{T T}\right)^{-1} .
$$

Taking the divergence of the product of these filtered maps, as prescribed in [32], gives,

$$
\frac{\kappa_{l}^{T T}}{A_{l}^{T T}}=-\int d^{2} \hat{\mathbf{n}} e^{-i \hat{\mathbf{n}} \cdot l}\left\{\nabla \cdot\left[\mathbf{G}^{T T}(\hat{\mathbf{n}}) L^{T}(\hat{\mathbf{n}})\right]\right\} .
$$

Here, the real-space lensing convergence field constructed from temperature data is

$$
\kappa^{T T}(\hat{\mathbf{n}})=\int \frac{d^{2} l}{(2 \pi)^{2}} e^{i l \cdot \hat{\mathbf{n}}} \kappa_{l}^{T T} .
$$

The normalization factor is given by

$$
\frac{1}{A_{l}^{T T}}=\frac{2}{l^{2}} \int \frac{d^{2} l_{1}}{(2 \pi)^{2}}\left[\boldsymbol{l} \cdot \boldsymbol{l}_{1}\right] W_{l_{1}}^{T T} W_{l_{2}}^{T} f^{T T}\left(\boldsymbol{l}_{1}, \boldsymbol{l}_{2}\right)
$$

with

$$
f^{T T}\left(\boldsymbol{l}_{1}, \boldsymbol{l}_{2}\right)=\left[\boldsymbol{l} \cdot \boldsymbol{l}_{1}\right] \tilde{C}_{l_{1}}^{T T}+\left[\boldsymbol{l} \cdot \boldsymbol{l}_{2}\right] \tilde{C}_{l_{2}}^{T T}
$$

and $\boldsymbol{l}=\boldsymbol{l}_{1}+\boldsymbol{l}_{2}$.

The mean of each reconstructed convergence stamp is set to zero to remove fluctuations on scales larger than the size of the stamp. Each reconstructed convergence stamp is then 

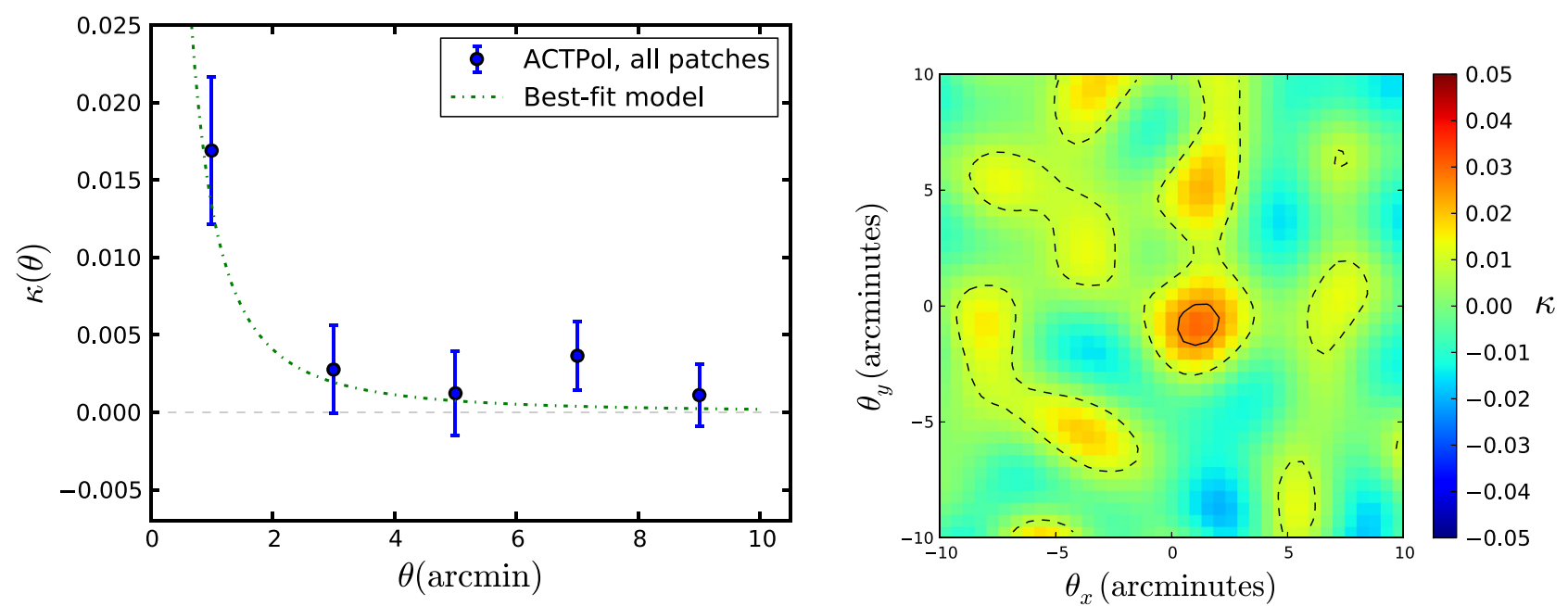

FIG. 1 (color online). Left: The azimuthally averaged signal from stacked reconstructed convergence stamps centered on CMASS galaxy positions for all three ACTPol deep fields combined. The green dashed curve shows the best-fit NFW profile. Right: The reconstructed convergence stack in the two-dimensional plane, where the horizontal and vertical scales are in arcminutes. We also show $1 \sigma$ (dashed) and $3 \sigma$ (solid) contours; the signal is the dark red spot in the middle. The peak is offset by about $1^{\prime}$ from the center; offsets of $>1^{\prime}$ are seen roughly $20 \%$ of the time in simulations of centered input halos given ACTPol noise levels. The detection significance above null is $3.8 \sigma$ within 10 arcminutes, and the best-fit curve from [50] is preferred over null with a significance of 3.2 $\sigma$ within 10 arcminutes.

low-pass filtered by setting modes with $l>5782$ to zero. This corresponds to ignoring modes smaller than the 1.4' beam scale.

The reconstructed lensing convergence stamps from a given ACTPol patch are then stacked (i.e., averaged). A "mean field" stamp needs to be subtracted from this stack since the apodization window does not leave the mean of the reconstructed stack identically zero in the absence of any signal $[53,54]$. We construct a mean field stamp from the average reconstruction of 15 realizations of random positions in the corresponding ACTPol patch. Each random-position realization has the same number of stamps as are in the galaxy stack. Thus, by construction, the meanfield-subtracted galaxy stacks show any excess signal above that from random locations.

In order to construct the covariance matrix for each patch, we construct 50 independent realizations of simulated ACTPol data for each patch. These simulations have noise and beam properties matched to the data and include only lensing by large-scale structure. We repeat the procedure performed on the data on each of the 50 independent simulations. The covariance matrix for each patch is then obtained by calculating the covariance of radial profiles across these 50 mean-field-subtracted, mean stamps. In this way, the covariance matrices capture the correlations between radial bins. This procedure also takes into account any additional covariance coming from overlapping stamps. In addition, it also folds in the uncertainty in the subtracted mean field. (Note that we use simulations to characterize the covariance matrix since stacking on random positions in the data does not capture the variance due to overlapping stamps and meanfield subtraction. A typical mean-field amplitude is 0.03 , and the uncertainty is $\approx 20 \%$ of the errorbars shown in Fig. 1.)

The pipeline described above is implemented for each ACTPol patch separately as well as for all the patches combined. The latter is done by stacking the three meanfield-subtracted galaxy stacks for each ACTPol data patch. The combined-patch covariance matrix is obtained by combining the 50 mean simulated convergence stamps for each patch and calculating the variance across all 150 mean stamps.

This pipeline is tested on a suite of simulations where $70^{\prime} \times 70^{\prime} \mathrm{CMB}$ stamps are lensed with Navarro-FrenkWhite (NFW) cluster profiles [55] with varying levels of instrument noise, beam resolution, and pixelization. The pipeline returns unbiased reconstructions (to $\approx 0.1 \sigma$ ) and $S / N$ estimates in agreement with previous analyses [32]. In particular, the expected detection significance stacking a sample of roughly 12000 galaxies in lensed CMB stamps with ACTPol beam and noise properties is $4.2 \sigma$. For this estimate, the masses, concentrations, and redshifts of the lensing galaxies are assumed to be the mean values of the CMASS subsample with optical weak lensing follow-up described above [50].

Results.-We show the result of the combined-patch stack of reconstructed convergence stamps centered on CMASS galaxies in Fig. 1. The left panel shows the measured azimuthally averaged lensing convergence profile, and the right panel shows the reconstructed lensing stack in the two-dimensional plane. We note that the signal peak in the two-dimensional plot is offset by about $1^{\prime}$. 
This is also seen in simulations of centered input halos given ACTPol noise levels, where offsets of $>1^{\prime}$ are seen roughly $20 \%$ of the time. We also note that this offset is well within the virial radius of CMASS halos. The profile has been binned, with inverse-variance weighting, in annuli that are four-pixels (2 arcminutes) wide so that correlations between neighboring bins in general do not exceed $50 \%$. The exceptions are that for the stacks on galaxy positions, the $3 \mathrm{rd}$ and 4 th bins are correlated by $65 \%$, and the 4th and 5 th bins are correlated by $70 \%$. This is due to overlapping stamps, as the galaxy locations are more correlated than random positions.

The significance of this detection above the null hypothesis, including measured points within 10 arcminutes of the profile center, is $3.8 \sigma$. This is calculated using the combined-patch covariance matrix, $\mathbf{C}$, where

$$
\left(\frac{S}{N}\right)^{2}=\chi_{\text {null }}^{2}=\sum_{\theta_{1}, \theta_{2} \leq 10^{\prime}} \kappa\left(\theta_{1}\right) \mathbf{C}^{-1} \kappa\left(\theta_{2}\right) .
$$

Restricting this to 4 arcminutes from the profile center, where most of the $S / N$ is from, gives a detection significance above null of $3.6 \sigma$.

We fit the data set within 10 arcminutes from the center with an NFW profile, which is the projected and redshiftaveraged mass density as in, e.g., [56]. We vary the mass and concentration and obtain a best-fit profile with a mass of $M_{200 \bar{\rho}_{0}}=(2.0 \pm 0.7) \times 10^{13} h^{-1} M_{\odot}$ and a concentration of $c_{200 \bar{\rho}}=(5.4 \pm 0.8)$. This result is obtained by imposing a prior on the $c-M$ relation from [57] assuming Gaussian errors on the normalization of this relation of $20 \%$ as found in [50]. We note that the best-fit mass and mass error are unchanged with and without the prior; however, since there is significant degeneracy in the concentration, given our noise levels, the prior influences the best-fit $c_{200 \bar{\rho}_{0}}$ and corresponding error. This best-fit curve gives a reduced chi-square of $\chi^{2} / \nu=1.5$ for $\nu=3$ degrees of freedom, and is consistent with the best-fit curve from [50]. The data also favor the best-fit curve from [50] over the null line $(\kappa=0)$ at a significance of $3.2 \sigma$ within 10 arcminutes, where we calculate this significance using $\sqrt{\chi_{\text {null }}^{2}-\chi_{\text {best-fit }}^{2}}$. Restricting to within 4 arcminutes, the model is favored over null with a significance of $2.9 \sigma$.

The profile of the reconstructed lensing stack for each ACTPol patch is shown in Fig. 2. An excess above null is seen in all three patches with a significance of $2.0 \sigma, 3.6 \sigma$, and $1.3 \sigma$ within 4 arcminutes for $D 1, D 5$, and D6, respectively. The black-dashed curve in Fig. 2 is an NFW profile with the best-fit mass and concentration found from optical weak lensing of a subset of the CMASS galaxy sample [50]. This best-fit mass and concentration for the subset is $M_{200 \bar{\rho}_{0}}=2.3 \times 10^{13} h^{-1} M_{\odot}$ and $c_{200 \bar{\rho}_{0}}=5.0$, where the concentration is from the best-fit concentrationmass relation found in [50], calculated at the mean redshift of the subset $(z=0.55)$. (In [50], a best-fit of $c_{200 \bar{\rho}_{0}}=5.0$ is

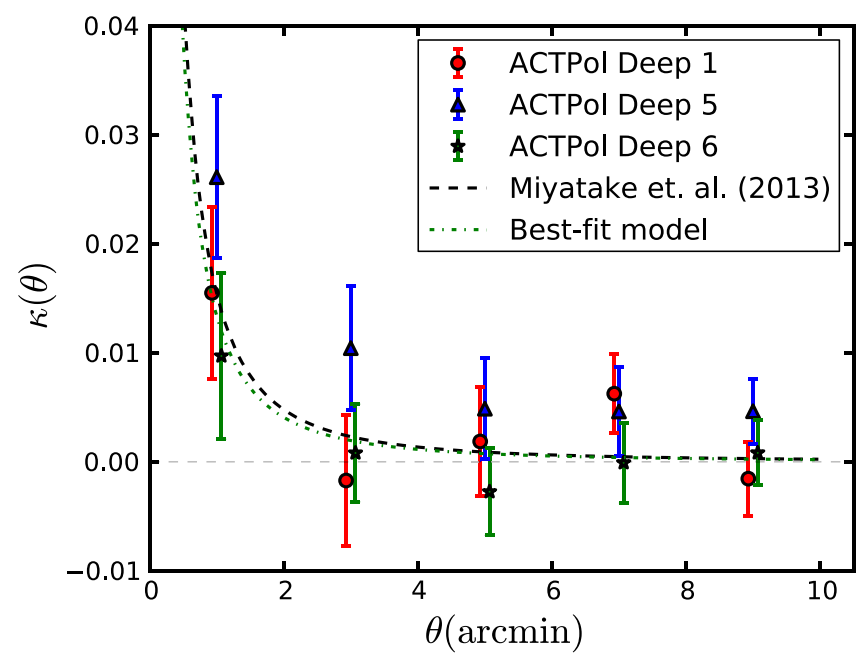

FIG. 2 (color online). Shown are reconstructed convergence profiles centered on CMASS galaxy positions for each ACTPol deep field separately. The significance with respect to null within 4 arcminutes is $2.0 \sigma, 3.6 \sigma$, and $1.3 \sigma$ for ACTPol Deep 1, 5, and 6, respectively. The green dashed curve is the best-fit NFW profile from all the Deep fields combined, and the black dashed curve is the best-fit NFW profile from a subset of the CMASS galaxies measured via optical weak lensing [50].

found for CMASS galaxies when their model allows for off centering of CMASS galaxies in dark matter halos. Without this degree of freedom, a best-fit of $c_{200 \bar{\rho}_{0}}=3.2$ is found.)

Systematic Checks.-Two different null tests are performed to verify the robustness of the signal. The first is to stack on random positions in the data. As mentioned above, all of the stacked images have a subtracted mean field stamp that is determined from averaging 15 realizations of randomly selected stamps from the data. Therefore, by construction, the measured signal is the excess above that from random locations. However, we show a single random-position realization which contains the same number of stamps as are in the galaxy stack. We subtract the mean field stamp from this single realization and plot the resulting profile in the top panel of Fig. 3 (brown circles). The data set is consistent with the null hypothesis with a probability to exceed (PTE) of 0.92 .

The second null test is a curl test where we repeat the analysis of stacking reconstructions centered on CMASS galaxies and subtract a mean field stamp as before. However, this time the divergence in Eq. (7) is replaced with a curl, and the first instance of the dot product $\boldsymbol{l} \cdot \boldsymbol{l}_{1}$ in Eq. (9) (not in $f^{T T}$ ) is replaced with a cross product $[4,58,59]$, where both the curl and cross product are projected perpendicular to the image plane. The reconstruction is then expected to contain only noise since lensing is not expected to generate a curl signal in temperature maps. The curl reconstruction data points scatter about zero, with a PTE of 0.08 , as shown in Fig. 3 (red stars). 

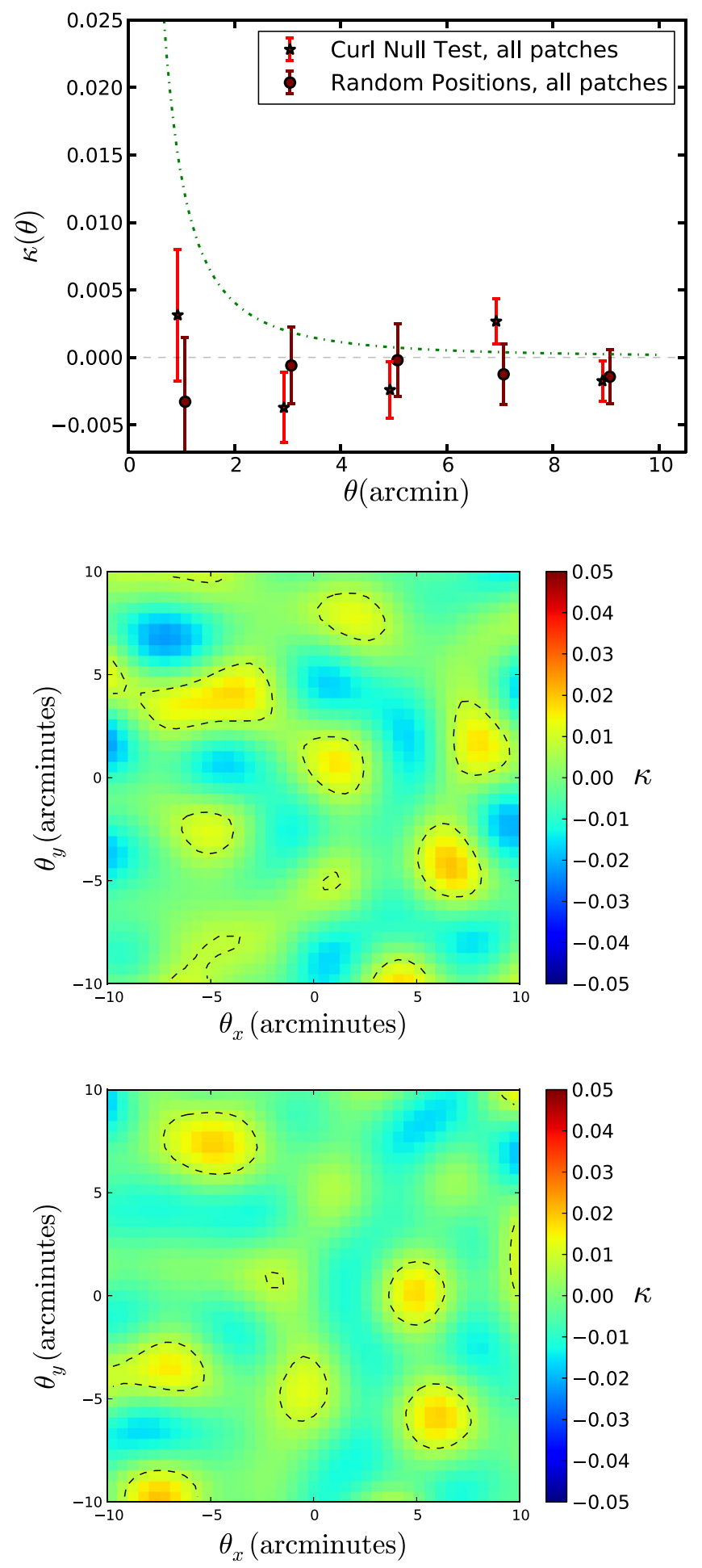

FIG. 3 (color online). Top panel: Shown are the curl null test performed on the stack of reconstructed convergence stamps centered on CMASS galaxy positions, and a randomposition null test where reconstructed convergence stamps are centered on random positions in the data. Middle and bottom panels: Shown are the curl and random-position null tests, respectively, in the two-dimensional plane. We also show 1 -sigma contours; the lack of a red spot in the middle confirms the null test.
As can be seen in Fig. 2, the mean signal is highest in D5. A histogram analysis of the stamps in both $D 5$ and in the quadrant of $D 5$ with the highest mean signal shows no apparent outliers. We note that excluding this quadrant from our analysis still results in a $S / N>3 \sigma$ within 10 arcminutes.

We also consider several possible contaminants that could bias a detection of CMB halo lensing. Ionized gas in clusters hosting the stacked galaxies could produce a decrement in the CMB temperature at $146 \mathrm{GHz}$ due to the thermal Sunyaev-Zeldovich (tSZ) effect $[60,61]$. In order to determine the effect of such a contaminant on the lensing reconstruction, we added a Gaussian decrement with a peak value of $-35 \mu \mathrm{K}$ and $1 \sigma$ width of 1 arcminute (the virial radius of a $10^{13} M_{\odot}$ halo at $z=0.6$ is roughly $1.5^{\prime}$ ) to CMB temperature maps lensed by NFW profiles as discussed above. We adopted this as a conservative level of tSZ for CMASS halos (see, for example, [62]). This contamination resulted in the reconstruction being biased low by about $0.3 \sigma$ within 3 arcminutes at ACTPol noise levels, with negligible bias beyond 3 arcminutes. An identical check was performed for $35 \mu \mathrm{K}$ increments (corresponding to point source emission) with a similar suppression of the signal. In addition, no appreciable tSZ decrement or point source increment is found when stacking the stamps taken directly from $\mathrm{CMB}$ temperature maps and centered on the CMASS galaxies, after these stamps have been filtered to isolate modes between $1000<l<8000$. These checks indicate that the detected positive signals in Figs. 1 and 2 do not arise from tSZ or point source emission. The kinetic SZ effect due to the bulk motion of the cluster will produce a similar symmetric increment or decrement. Furthermore, asymmetric contaminants, like those due to the kinetic SZ effect from internal gas motions, do not coherently align with the CMB gradient and only add noise by construction of the estimator.

The stacked lensing convergence measured in Figs. 1 and 2 could also have contributions that are not due to CMB lensing by the halo that each galaxy resides in (the 1-halo term), but instead are due to correlated halos in the vicinity of the galaxies (the 2-halo term, $[63,64]$ ). Since most of our detected signal is within a 2 arcminute region, where the 1-halo term dominates over the 2-halo term (see, for example, Fig. 7 in [50]), one would not expect the 2-halo term to contribute significantly to the detection significance in this Letter.

Discussion.-We have presented the stacked reconstructed lensing convergence of CMASS galaxies within the first season ACTPol deep fields and shown evidence of $\mathrm{CMB}$ lensing from these halos at a significance of $3.8 \sigma$ above null. The lensing convergence is directly related to the projected density profile of these halos and hence, our results demonstrate that it is possible to constrain the mass profile of massive objects using $\mathrm{CMB}$ lensing alone. 
We find a best-fit mass and concentration from the stacked convergence stamps of $M_{200 \bar{\rho}_{0}}=(2.0 \pm 0.7) \times$ $10^{13} h^{-1} M_{\odot}$ and $c_{200 \bar{\rho}}=(5.4 \pm 0.8)$ fitting to an NFW profile. These mass and concentration values are in broad agreement with the optical weak lensing estimates in [50] based on a subset of the CMASS galaxy sample. Our data also favors the best-fit profile from [50] over a null line at a significance of $3.2 \sigma$ within 10 arcminutes.

With this Letter, we demonstrate that CMB observations are now achieving the sensitivity and resolution to provide mass estimates of dark matter halos belonging to galaxy groups and clusters. With the advent of next-generation CMB surveys, we expect this technique to be further exploited, thus opening a new window on the dark Universe.

The authors would like to thank Hironao Miyatake, Surhud More, and Anze Slosar for useful discussions regarding CMASS and BOSS galaxies. MM acknowledges support from an SBU-BNL Research Initiatives Seed Grant: Grant No. 37298, Project No. 1111593. This work was supported by the U.S. National Science Foundation through Grants No. AST-0408698 and No. AST-0965625 for the ACT project, as well as Grants No. PHY-0855887 and No. PHY-1214379. Funding was also provided by Princeton University, the University of Pennsylvania, Cornell University, and a Canada Foundation for Innovation (CFI) Grant to UBC. ACT operates in the Parque Astronómico Atacama in northern Chile under the auspices of the Comisión Nacional de Investigación Científica y Tecnológica de Chile (CONICYT). Computations were performed on the GPC supercomputer at the SciNet HPC Consortium. SciNet is funded by the CFI under the auspices of Compute Canada, the Government of Ontario, the Ontario Research FundResearch Excellence; and the University of Toronto. The development of multichroic detectors and lenses was supported by NASA Grants No. NNX13AE56G and No. NNX14AB58G. Funding from ERC Grant No. 259505 supports SN, JD, and TL. RD was supported by CONICYT Grants No. QUIMAL-120001 and No. FONDECYT1141113. We gratefully acknowledge support from the Misrahi and Wilkinson research funds.

[1] K. M. Smith, O. Zahn, and O. Doré, Phys. Rev. D 76, 043510 (2007).

[2] C. M. Hirata, S. Ho, N. Padmanabhan, U. Seljak, and N. A. Bahcall, Phys. Rev. D 78, 043520 (2008).

[3] S. Das, T. A. Marriage, P. A. R. Ade, P. Aguirre, M. Amiri, J. W. Appel, L. F. Barrientos, E. S. Battistelli, J. R. Bond, B. Brown et al., Astrophys. J. 729, 62 (2011).

[4] A. van Engelen, R. Keisler, O. Zahn, K. A. Aird, B. A. Benson, L. E. Bleem, J. E. Carlstrom, C. L. Chang, H. M. Cho, T. M. Crawford et al., Astrophys. J. 756, 142 (2012).

[5] Planck Collaboration, Astron. Astrophys. 571, A17 (2014).
[6] B. D. Sherwin, S. Das, A. Hajian, G. Addison, J. R. Bond, D. Crichton, M. J. Devlin, J. Dunkley, M. B. Gralla, M. Halpern et al., Phys. Rev. D 86, 083006 (2012).

[7] L. E. Bleem, A. van Engelen, G. P. Holder, K. A. Aird, R. Armstrong, M. L. N. Ashby, M. R. Becker, B. A. Benson, T. Biesiadzinski, M. Brodwin et al., Astrophys. J. 753, L9 (2012).

[8] J. E. Geach, R. C. Hickox, L. E. Bleem, M. Brodwin, G. P. Holder, K. A. Aird, B. A. Benson, S. Bhattacharya, J. E. Carlstrom, C. L. Chang et al., Astrophys. J. Lett. 776, L41 (2013).

[9] G. P. Holder, M. P. Viero, O. Zahn, K. A. Aird, B. A. Benson, S. Bhattacharya, L. E. Bleem, J. Bock, M. Brodwin, J. E. Carlstrom et al., Astrophys. J. Lett. 771, L16 (2013).

[10] Planck Collaboration, Astron. Astrophys. 571, A18 (2014).

[11] D. Hanson, S. Hoover, A. Crites, P. A. R. Ade, K. A. Aird, J. E. Austermann, J. A. Beall, A. N. Bender, B. A. Benson, L. E. Bleem et al., Phys. Rev. Lett. 111, 141301 (2013).

[12] P. A. R. Ade, Y. Akiba, A. E. Anthony, K. Arnold, M. Atlas, D. Barron, D. Boettger, J. Borrill, C. Borys, S. Chapman et al., Phys. Rev. Lett. 112, 131302 (2014).

[13] P. A. R. Ade, Y. Akiba, A. E. Anthony, K. Arnold, D. Barron, D. Boettger, J. Borrill, S. Chapman, Y. Chinone, M. Dobbs et al., Phys. Rev. Lett. 113, 021301 (2014).

[14] N. Hand, A. Leauthaud, S. Das, B. D. Sherwin, G. E. Addison, J. R. Bond, E. Calabrese, A. Charbonnier, M. J. Devlin, J. Dunkley et al., arXiv:1311.6200 [Phys. Rev. D (to be published)].

[15] F. Bianchini, P. Bielewicz, A. Lapi, J. Gonzalez-Nuevo, C. Baccigalupi, G. de Zotti, L. Danese, N. Bourne, A. Cooray, L. Dunne et al., arXiv:1410.4502 [Astrophys. J. (to be published)].

[16] M. A. DiPompeo, A. D. Myers, R. C. Hickox, J. E. Geach, G. Holder, K. N. Hainline, and S. W. Hall, Mon. Not. R. Astron. Soc. 446, 3492 (2015).

[17] N. Fornengo, L. Perotto, M. Regis, and S. Camera, arXiv: 1410.4997.

[18] J. C. Hill and D. N. Spergel, J. Cosmol. Astropart. Phys. 02 (2014) 030.

[19] Planck Collaboration, Astron. Astrophys. 571, A16 (2014).

[20] K. N. Abazajian, K. Arnold, J. Austermann, B. A. Benson, C. Bischoff, J. Bock, J. R. Bond, J. Borrill, E. Calabrese, J. E. Carlstrom et al., Astropart. Phys. 63, 66 (2015).

[21] K. N. Abazajian, K. Arnold, J. Austermann, B. A. Benson, C. Bischoff, J. Bock, J. R. Bond, J. Borrill, I. Buder, D. L. Burke et al., Astropart. Phys. 63, 55 (2015).

[22] E. Calabrese, R. Hložek, N. Battaglia, J. R. Bond, F. de Bernardis, M. J. Devlin, A. Hajian, S. Henderson, J. C. Hil, A. Kosowsky et al., J. Cosmol. Astropart. Phys. 08 (2014) 010 .

[23] U. Seljak and M. Zaldarriaga, Astrophys. J. 538, 57 (2000).

[24] M. Zaldarriaga, Phys. Rev. D 62, 063510 (2000).

[25] S. Dodelson and G. D. Starkman, arXiv:astro-ph/0305467.

[26] G. Holder and A. Kosowsky, Astrophys. J. 616, 8 (2004).

[27] S. Dodelson, Phys. Rev. D 70, 023009 (2004).

[28] C. Vale, A. Amblard, and M. White, New Astron. 10, 1 (2004). 
[29] M. Maturi, M. Bartelmann, M. Meneghetti, and L. Moscardini, Astron. Astrophys. 436, 37 (2005).

[30] A. Lewis and L. King, Phys. Rev. D 73, 063006 (2006).

[31] W. Hu, D. E. Holz, and C. Vale, Phys. Rev. D 76, 127301 (2007).

[32] W. Hu, S. DeDeo, and C. Vale, New J. Phys. 9, 441 (2007).

[33] J. Yoo and M. Zaldarriaga, Phys. Rev. D 78, 083002 (2008).

[34] J. Yoo, M. Zaldarriaga, and L. Hernquist, Phys. Rev. D 81, 123006 (2010).

[35] J.-B. Melin and J. G. Bartlett, arXiv:1408.5633

[36] D. J. Eisenstein, D. H. Weinberg, E. Agol, H. Aihara, C. A. Prieto, S. F. Anderson, J. A. Arns, É. Aubourg, S. Bailey, E. Balbinot et al., Astron. J. 142, 72 (2011).

[37] K. S. Dawson, D. J. Schlegel, C. P. Ahn, S. F. Anderson, É. Aubourg, S. Bailey, R. H. Barkhouser, J. E. Bautista, A. Beifiori, A. A. Berlind et al., Astron. J. 145, 10 (2013).

[38] C. P. Ahn, R. Alexandroff, C. Allende Prieto, F. Anders, S. F. Anderson, T. Anderton, B. H. Andrews, É. Aubourg, S. Bailey, F. A. Bastien et al., Astrophys. J. Suppl. Ser. 211, 17 (2014).

[39] M. D. Niemack, P. A. R. Ade, J. Aguirre, F. Barrientos, J. A. Beall, J. R. Bond, J. Britton, H. M. Cho, S. Das, M. J. Devlin et al., Proc. SPIE Int. Soc. Opt. Eng. 7741, 77411S 2010).

[40] S. Naess, M. Hasselfield, J. McMahon, M. D. Niemack, G. E. Addison, P. A. R. Ade, R. Allison, M. Amiri, N. Battaglia, J. A. Beall et al., J. Cosmol. Astropart. Phys. 10 (2014) 007.

[41] Planck Collaboration, Astron. Astrophys. 571, A1 (2014).

[42] T. Louis, G. E. Addison, M. Hasselfield, J. R. Bond, E. Calabrese, S. Das, M. J. Devlin, J. Dunkley, R. Dünner, M. Gralla et al., J. Cosmol. Astropart. Phys. 7 (2014) 016.

[43] D. G. York, J. Adelman, J. E. Anderson Jr, S. F. Anderson, J. Annis, N. A. Bahcall, J. Bakken, R. Barkhouser, S. Bastian, E. Berman et al., Astron. J. 120, 1579 (2000).

[44] J. E. Gunn, W. A. Siegmund, E. J. Mannery, R. E. Owen, C. L. Hull, R. F. Leger, L. N. Carey, G. R. Knapp, D. G. York, W. N. Boroski et al., Astron. J. 131, 2332 (2006).

[45] D. J. Eisenstein, J. Annis, J. E. Gunn, A. S. Szalay, A. J. Connolly, R. C. Nichol, N. A. Bahcall, M. Bernardi, S. Burles, F. J. Castander et al., Astron. J. 122, 2267 (2001).

[46] L. Anderson, E. Aubourg, S. Bailey, D. Bizyaev, M. Blanton, A. S. Bolton, J. Brinkmann, J. R. Brownstein, A. Burden, A. J. Cuesta et al., Mon. Not. R. Astron. Soc. 427, 3435 (2012).
[47] B. A. Reid, L. Samushia, M. White, W. J. Percival, M. Manera, N. Padmanabhan, A. J. Ross, A. G. Sánchez, S. Bailey, D. Bizyaev et al., Mon. Not. R. Astron. Soc. 426, 2719 (2012).

[48] https://data.sdss3.org/datamodel/files/SPECTRO_REDUX/ specObj.html. We used the keywords BOSS_TARGET1\&\&2, SPECPRIMARY $==1, \quad$ ZWARNING_NOQSO $==0$, and (CHUNK! ="boss1")\&\&(CHUNK! ="boss2"). The keywords are described here: https://www.sdss3.org/dr10/ algorithms/boss_galaxy_ts.php.

[49] http://data.sdss3.org/datamodel/files/BOSS_PHOTOOBJ/ RERUN/RUN/CAMCOL/photoObj.html.

[50] H. Miyatake, S. More, R. Mandelbaum, M. Takada, D. Spergel, J.-P. Kneib, D. P. Schneider, J. Brinkmann, J. R. Brownstein et al., arXiv:1311.1480.

[51] C. Heymans, L. Van Waerbeke, L. Miller, T. Erben, H. Hildebrandt, H. Hoekstra, T. D. Kitching, Y. Mellier, P. Simon, C. Bonnett et al., Mon. Not. R. Astron. Soc. 427, 146 (2012).

[52] W. Hu and T. Okamoto, Astrophys. J. 574, 566 (2002).

[53] D. Hanson, G. Rocha, and K. Górski, Mon. Not. R. Astron. Soc. 400, 2169 (2009).

[54] T. Namikawa, D. Hanson, and R. Takahashi, Mon. Not. R. Astron. Soc. 431, 609 (2013).

[55] J. F. Navarro, C. S. Frenk, and S. D. White, Astrophys. J. 490, 493 (1997).

[56] M. Bartelmann, Astron. Astrophys. 313, 697 (1996).

[57] A. V. Macciò, A. A. Dutton, F. C. van den Bosch, B. Moore, D. Potter, and J. Stadel, Mon. Not. R. Astron. Soc. 378, 55 (2007).

[58] A. Cooray, M. Kamionkowski, and R. R. Caldwell, Phys. Rev. D 71, 123527 (2005).

[59] B. D. Sherwin, S. Das, A. Hajian, G. Addison, J. R. Bond, D. Crichton, M. J. Devlin, J. Dunkley, M. B. Gralla, M. Halpern et al., Phys. Rev. D 86, 083006 (2012).

[60] R. A. Sunyaev and Y. B. Zel'dovich, Comments Astrophys. Space Phys. 2, 66 (1970).

[61] R. A. Sunyaev and Y. B. Zel'dovich, Comments Astrophys. Space Phys. 4, 173 (1972).

[62] N. Hand, J. W. Appel, N. Battaglia, J. R. Bond, S. Das, M. J. Devlin, J. Dunkley, R. Dünner, T. Essinger-Hileman, J. W. Fowler et al., Astrophys. J. 736, 39 (2011).

[63] C.-P. Ma and J. N. Fry, Astrophys. J. 543, 503 (2000).

[64] U. Seljak, Mon. Not. R. Astron. Soc. 318, 203 (2000). 